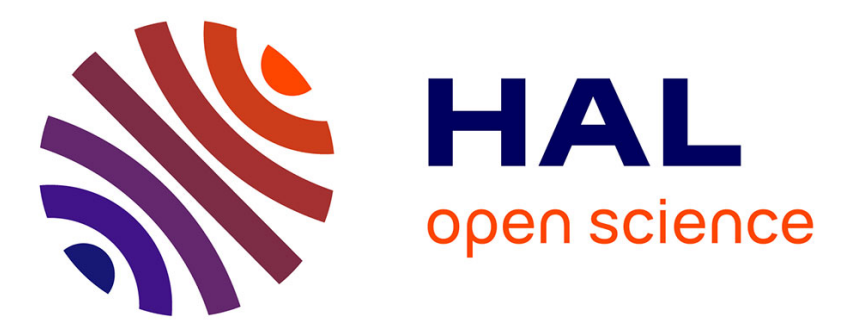

\title{
Que nous apprennent les BRIC? Trois conjectures sur les économies émergentes
}

Julien Vercueil

\section{To cite this version:}

Julien Vercueil. Que nous apprennent les BRIC? Trois conjectures sur les économies émergentes. Mondes en Développement, 2012, 158, pp.25 - 25. 10.3917/med.158.0025 . halshs-01420397

\section{HAL Id: halshs-01420397 https://shs.hal.science/halshs-01420397}

Submitted on 20 Dec 2016

HAL is a multi-disciplinary open access archive for the deposit and dissemination of scientific research documents, whether they are published or not. The documents may come from teaching and research institutions in France or abroad, or from public or private research centers.
L'archive ouverte pluridisciplinaire HAL, est destinée au dépôt et à la diffusion de documents scientifiques de niveau recherche, publiés ou non, émanant des établissements d'enseignement et de recherche français ou étrangers, des laboratoires publics ou privés.

\section{(ㅇ)(1) $\$$}

Distributed under a Creative Commons Attribution - NonCommercial - NoDerivatives 44.0 


\title{
Que nous apprennent les « BRIC » ?
}

\section{Trois conjectures sur les économies émergentes}

\author{
Julien Vercueil \\ CEMI (EHESS), Université de Lyon
}

L'expression " marchés émergents " a été forgée il y a trente ans. Depuis sa création, elle a donné naissance à des expressions dérivées, comme "économies à marchés émergents ", "économies émergentes ", "BRIC », et d'autres encore. Toutefois, les phénomènes qui se trouvent à la base du processus d'émergence économique n'ont pas encore fait l'objet d'une analyse comparative en profondeur. La notion même "d'économies émergentes " n'est pas tout à fait clarifiée, comme en attestent les sens variés que leur attribuent différentes publications officielles contemporaines (IMF, 2010)

Ce texte propose de partir d'une définition exploratoire de la notion " d'économie émergente " pour s'intéresser aux quatre plus éminentes d'entre elles: le Brésil, la Russie, l'Inde et la Chine. Ces économies seront qualifiées "d'émergentes" en tant qu'économies à revenus intermédiaires connaissant un processus de transformation économique et institutionnelle qui se traduit par une forte croissance économique et une participation accrue aux courants d'échanges mondiaux.

La notion de "revenu intermédiaire " est ici définie par un PIB par habitant en dollars de parités de pouvoir d'achat compris entre $10 \%$ et $75 \%$ de la moyenne des pays riches; la " forte croissance économique " implique un taux de croissance annuel moyen du PIB supérieur à celui des pays riches dans la dernière décennie ; la participation accrue aux courants d'échanges mondiaux est mesurée par l'écart positif entre les croissances du commerce extérieur de biens et services et des IDE sortants d'une part, et celle du PIB d'autre part; quant aux transformations économiques et institutionnelles, elles relèvent d'une analyse qualitative qui s'intéresse à la manière dont le régime juridique des activités économiques a été transformé durant les dernières décennies dans ces pays et à son influence effective sur les modes de coordination et les comportements économiques observables dans les pays considérés, notamment - mais pas exclusivement - dans les relations entre les agents résidents et le reste du monde.

Sous cette acception, une soixantaine d'économies peuvent actuellement être qualifiées d'émergentes. Elles représentent une population de plus de quatre milliards d'habitants, répartis sur tous les continents du globe. Notre approche de la notion suppose que les «BRIC » peuvent constituer une illustration intéressante de ce groupe vaste et hétérogène de pays en développement partageant une expérience de transformations accélérées.

L'objet de cette contribution est de présenter trois conjectures à propos les "BRIC ", qui peuvent servir de base à des recherches plus approfondies. 


\section{Première conjecture. La cohérence institutionnelle est un préalable à l'émergence économique}

La question du rôle des institutions ${ }^{1}$ dans les transformations économiques des pays en développement et des pays à économie anciennement planifiée a fait l'objet d'une abondante littérature, depuis le début des années 1990 jusqu'à aujourd'hui. Initialement minoré par la théorie standard $^{2}$, le rôle joué par les institutions dans la manière dont les prescriptions de politique économique sont assimilées dans l'économie a progressivement été reconnu par une majorité de chercheurs, notamment dans les cas de la transition économique à l'Est (North, 1997, Stiglitz , 1999, Roland, 2000). La question posée ici est celle de savoir s'il existe des conditions institutionnelles de possibilité au déploiement de processus caractéristiques de l'émergence (en particulier, l'accélération durable de la croissance économique), et dans quelle mesure l'expérience des BRIC nous aide à cerner la nature de ces conditions.

La théorie de la régulation propose la notion de "complémentarité institutionnelle " pour caractériser l'importance des relations organiques entre les différentes institutions d'une économie (Boyer, 2003). Le terme de " cohérence institutionnelle " que nous utiliserons ici est proche de cette notion. II permet en outre d'intégrer le fait que certaines institutions peuvent être redondantes, donc non complémentaires au sens premier de ce terme, tout en exerçant, par leur agencement, une influence significative sur les comportements économiques. La cohérence institutionnelle s'exprime par le fait qu'un ensemble donné d'institutions, pour agir efficacement sur les comportements, doit produire des incitations susceptibles d'entrer en contradiction mutuelle dans un nombre de cas aussi limité que possible (Vercueil, 2002).

Notre approche part de l'idée que le canevas institutionnel d'une économie est un ensemble enchevêtré d'institutions de niveaux différents qui exercent leurs effets sur plusieurs échelles de temps et d'espace, posant donc a priori des problèmes de cohérence. L'enchevêtrement institutionnel est doublé de l'hétérogénéité des agents constitutifs d'une économie, qui fait que la cohérence d'un ensemble d'institutions donné peut toujours être contestée, puisqu'un ensemble d'institutions qui exercerait de manière uniforme son pouvoir régulateur pourrait tout de même produire des incitations incohérentes sur les agents, si ces derniers étaient situés dans des positions différentes vis-à-vis d'elle. La prise en compte de la dimension temporelle du problème conduit à reconnaître aussi qu'une même institution, fonctionnant de manière constante dans le temps, est susceptible de produire sur le même groupe d'agents des incitations différentes à deux moments différents, notamment parce que d'autres conditions influant sur leurs décisions peuvent avoir changé dans l'intervalle.

On voit par là que la cohérence d'un ensemble d'institutions est toujours imparfaite, toujours remise en cause et toujours renouvelée. Du point de vue conceptuel, c'est donc aussi une notion ouverte : la cohérence d'un ensemble institutionnel donné répond à un domaine de validité relativement large, et non pas à des conditions strictement définies.

\footnotetext{
${ }^{1}$ Pour éviter de rentrer dans des discussions techniques, nous adopterons dans ce texte le sens donné par Douglas North au terme " institutions " comme "règles du jeu économique " (North, 1991). Les institutions peuvent être formelles, c'est à dire sanctionnées par des textes officiels tels que les lois et règlements, ou informelles, comme les habitudes collectives ou les conventions.

${ }^{2}$ La théorie standard est comprise ici dans le sens qui lui a été donné par Olivier Favereau (Favereau, 1989).
} 
Pour l'analyse de l'émergence économique, la difficulté de maniement du concept de cohérence (mais aussi de celui de complémentarité) institutionnelle est double : compte tenu de son caractère flexible, ne peut-on pas toujours trouver a posteriori une cohérence institutionnelle dans une économie qui a fortement progressé, si on la cherche avec suffisamment de conviction? Inversement, comment s'assurer a priori qu'un ensemble donné d'institutions puisse être doté d'une cohérence institutionnelle suffisante, pour un objet aussi complexe qu'une économie nationale?

Nous nous proposons d'illustrer l'idée de cohérence institutionnelle par l'étude comparative de certaines caractéristiques des comparées des BRIC dans les dernières décennies, tout en essayant d'éviter à la fois de procéder une reconstruction artificielle a posteriori et de donner l'impression d'un déterminisme institutionnel qui n'existe pas. L'idée que nous souhaitons défendre ici est que l'émergence économique n'a été rendue possible dans ces pays qu'une fois que institutions ont acquis un certain niveau de cohérence. Dans chaque cas, s'est dégagé parmi les acteurs publics et privés ce que l'on pourrait appeler un " accord critique ", explicite ou non, sur les priorités à donner à l'action publique et à l'activité de chacun qui a permis d'initier un mouvement cohérent de transformations économiques appelé à durer.

En Chine, la fin de l'ère Mao a rendu possible l'arrêt des coups de boutoir idéologiques dont le Parti communiste usait périodiquement pour se maintenir au pouvoir. Mais encore fallait-il que ses successeurs offrissent de nouvelles perspectives à la population. La mise en mouvement coordonnée de certaines institutions fondamentales du monde rural (comme le système de responsabilité des ménages, puis celui des entreprises et des administrations locales) a permis d'engager les campagnes dans la voie de sortie de l'extrême pauvreté, ouvrant la voie à de nouvelles transformations engagées par la suite sur le terrain économique (Lin et alii, 1996).

Pour le Brésil, après une première tentative manquée dans les années 1970 et la disparition de la dictature en 1985, la cohérence institutionnelle de la deuxième moitié des années 1990 s'est établie autour de la priorité à donner à l'éradication de l'inflation ouverte (Feijo et alii, 2009), qui affaiblissait la légitimité de l'État. Une fois l'étape franchie, les autorités ont acquis et conservé un niveau de crédit suffisant pour leur permettre de prolonger les transformations économiques et institutionnelles. L'alternance politique des années 2000, dans le contexte d'un fonctionnement moins conflictuel de la démocratie que dans la période précédente, ne s'est pas traduite par une inversion, mais par une adaptation des priorités du gouvernement (Bresser-Pereira, 2002).

Au début des années 1990, I'Inde avait devant elle l'exemple de l'extraordinaire essor de la Chine et d'autres pays asiatiques, pourtant partis de situations encore plus précaires que la sienne, et dont il lui était possible de tirer des enseignements; toutefois, la tâche des autorités indiennes était politiquement plus difficile qu'en Chine car les transformations devaient se faire à un rythme compatible avec la démocratie. La décennie 1990 se caractérise ainsi en Inde par une forme de constance des priorités accordées à certaines transformations clef (en particulier la décentralisation des décisions économiques), malgré une alternance qui continuait de s'exercer au Congrès (Ahluwalia, 2002).

Enfin, la Russie présente un cas particulier du point de vue de l'évolution des institutions et de leur rôle dans les processus de l'émergence économique. La décomposition des institutions du communisme, à la fin des années 1980, ouvre la possibilité aux autorités de la nouvelle Russie, 
bénéficiant d'une forte légitimité, de proposer un contrat social refondé à la population (Alexashenko, 1992, Dunlop, 1999). Ce n'est pas ce qui se produit. Au contraire, la décomposition et l'incohérence institutionnelles s'accélèrent dans la décennie qui suit. Les institutions formelles, qui se multiplient dans une grande confusion, n'ont pas de lien stable avec les institutions informelles qui se développent à grande vitesse de manière largement autonome. Dans le domaine monétaire, le rouble se trouve peu à peu subverti par le développement des impayés, des encaisses en dollars, du recours au troc ou à des substituts monétaires locaux qui finissent par affaiblir l'État lui-même en contribuant à le priver de ressources. Ce n'est qu'après le défaut d'août 1998 que les conditions $d^{\prime}$ 'une remise en cohérence des institutions sont à nouveau réunies par les autorités politiques : la période d'accélération de la Russie est donc aussi celle du retour de l'État et de ses institutions dans l'économie, dont ils avaient fini par presque disparaître durant la période précédente (Pagé et Vercueil, 2004).

\section{Deuxième conjecture. Les trajectoires réussies sont pilotées en temps réel}

La cohérence institutionnelle est loin de suffire à l'émergence : un faisceau d'institutions donné peut se trouver peu à peu dépassé par les mouvements économiques internes ou externes qui le rendent inopérant. Les conditions qui rendent possible la sortie de la grande pauvreté ne sont pas celles de la montée en gamme industrielle, ni celles qui favorisent le succès de la tertiarisation et de la progression vers une économie fondée sur la connaissance. Or, les changements opérés dans les pays émergents, pour produire des effets puissants, doivent se poursuivre sur plus d'une décennie. Une approche en termes de " big bang ", voulant du passé faire table rase et faire surgir un système économique moderne tout armé du front des économistes, paraît donc vouée à l'échec (Economic Commission for Europe, 1992). Les stratégies gagnantes semblent bien plutôt celles des grands trajets accomplis à petits pas (Vercueil, 2010).

De fait, les politiques menées dans les BRIC ne se sont pas caractérisées, pour celles qui ont connu un minimum d'efficacité, par l'application rigide d'un plan préconçu. Au contraire, elles se sont caractérisées par la malléabilité : les premières politiques menées ont été progressivement abandonnées à mesure que les conditions de leur efficacité s'affaiblissaient, tandis que de nouveaux outils étaient développés pour les remplacer. De ce point de vue, la démocratie recèle a priori un certain avantage sur les régimes autocratiques, en favorisant le renouvellement des positions via les élections. Mais la réussite économique de régimes autocratiques comme celui de la Chine montre que la démocratie, s'il est le plus souhaitable d'un point de vue éthique, n'est pas le seul système politique qui ait permis une mise en oeuvre évolutionnaire, parfois appelée « pragmatique » dans la presse, d'un ensemble de réformes économiques doté d'une certaine efficacité.

En Chine, les autorités ont eu tendance à tester d'abord les réformes dans des zones délimitées (les "zones économiques spéciales » par exemple) pour, après évaluation des résultats, les généraliser. Parfois ces réformes sont inspirées de l'extérieur, parfois elles sont déduites de l'observation des acteurs de terrain eux-mêmes; mais on serait bien en peine d'en trouver qui proviennent de manuels d'économie politique classique. Dans un cadre politique démocratique, les autorités indiennes ont elles aussi démontré leur aptitude à adapter le rythme des changements institutionnels impulsés aux contraintes du moment. En 1991, le choix est fait de démanteler en priorité le système d'autorisations administratives d'activités à l'exportation en priorité, puis d'éliminer ce système de licences l'industrie nationale, dans une séquence politique qui doit 
composer en avec un Congrès hostile à toute réforme radicale. Les privatisations n'interviennent que trois ans après le début du démantèlement du "Licence raj ", et progressent toujours à un rythme plus lent qu'annoncé ; la convertibilité de la roupie pour les transactions courantes n'est effective qu'au printemps 1994. Le cas russe présente une succession de phases bien particulière : après avoir tenté, durant les années 1990, de plaquer sur sa propre réalité des modèles théoriques conçus, sur des bases fragiles, pour d'autres contextes et qui devaient en quelques mois permettre de résorber des dysfonctionnements accumulés durant les soixante-dix années précédentes, la Russie a fini par rejeter ces modèles pour construire sa propre trajectoire de sortie de crise. Celle-ci s'est d'abord appuyée sur la dévaluation d'août 1998 et le défaut partiel sur les obligations de paiement extérieur, qui permettaient le desserrement de la contrainte monétaire et le redémarrage de la production domestique. Elle a ensuite été centrée sur la reprise en main de la fiscalité et du secteur énergétique par l'État et le décollage des prix des hydrocarbures. Au Brésil, la stabilisation monétaire de 1995 a été obtenue par l'application d'un plan approuvé par le FMI. Mais par la suite, les dirigeants brésiliens se sont démarqués des modèles fournis par les institutions financières internationales pour piloter la trajectoire de leur économie de manière pragmatique, en instaurant par exemple des programmes sociaux propres (système unifié d'assurance médicale gratuite à la fin des années 1990, bourse famille en 2002) qui ont pour objectif de corriger les déséquilibres constatés dans le développement de la société brésilienne.

Dans le même temps, les autorités de ces pays admettent l'idée que l'émergence économique s'accommode mal de désajustements persistants et importants de certaines variables-clef de la politique économique conjoncturelle : l'inflation, les taux d'intérêt, le déficit des finances publiques, l'endettement de l'État, les soldes des échanges extérieurs et le taux de change de la monnaie nationale ont été ainsi surveillés et maintenus dans des limites qui ont été considérées comme raisonnables, mais qui s'avèrent variables d'un pays à l'autre. L'émergence économique, comme tout processus de transformation rapide, est génératrice de déséquilibres multiples. Mais en ce qui concerne les BRIC, tout se passe comme si les autorités maintenaient ces déséquilibres dans un intervalle jugé acceptable par elles. Les graphiques 1, 2 et 3 illustrent sommairement cette idée en s'intéressant à trois indicateurs : les prix (et l'inflation), le budget de l'État (et son déficit) et la balance courante (et son déficit). 
Graphique 1.

Taux d'inflation comparés (\% de variation annuelle de
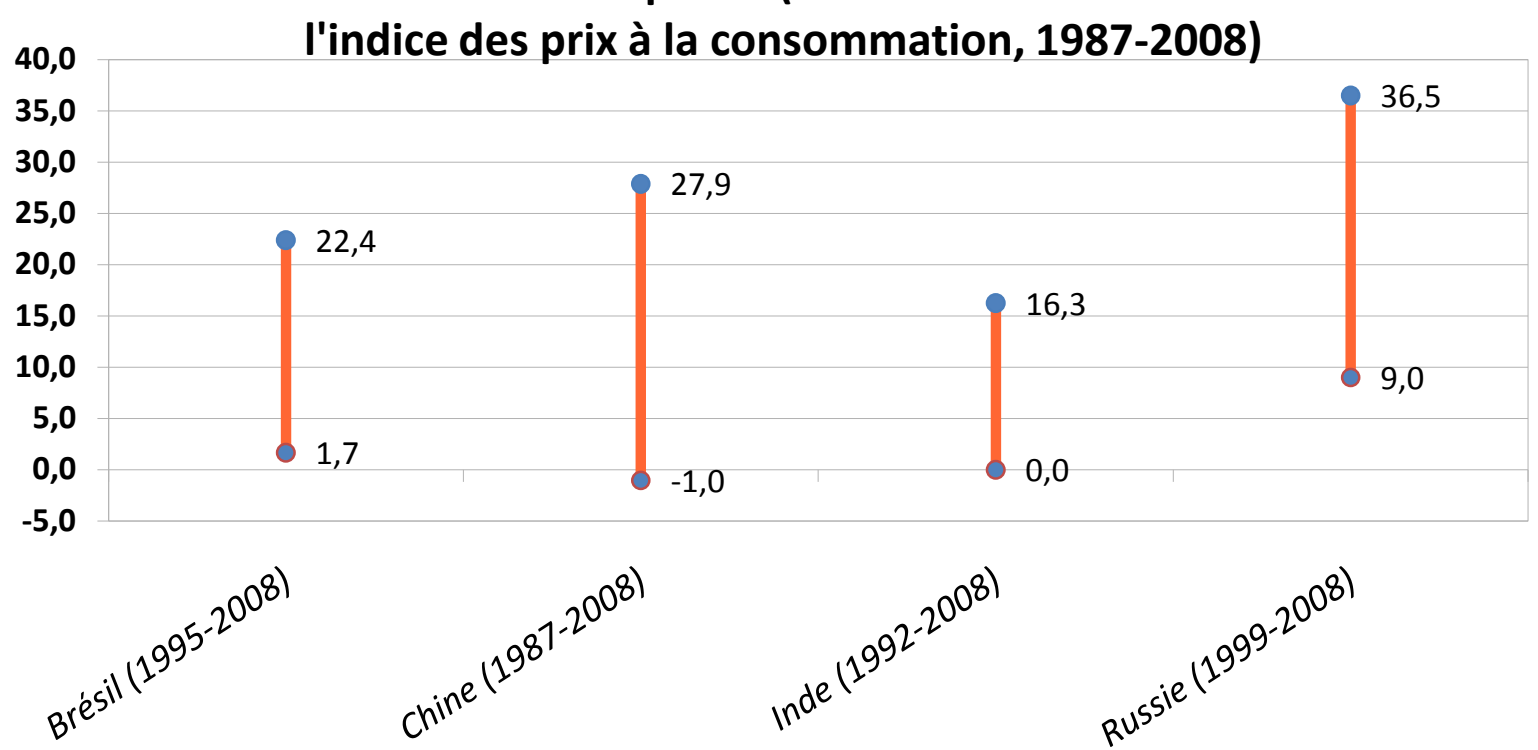

Graphique 2.

Soldes budgétaires comparés (\% PIB, 1995-2008)
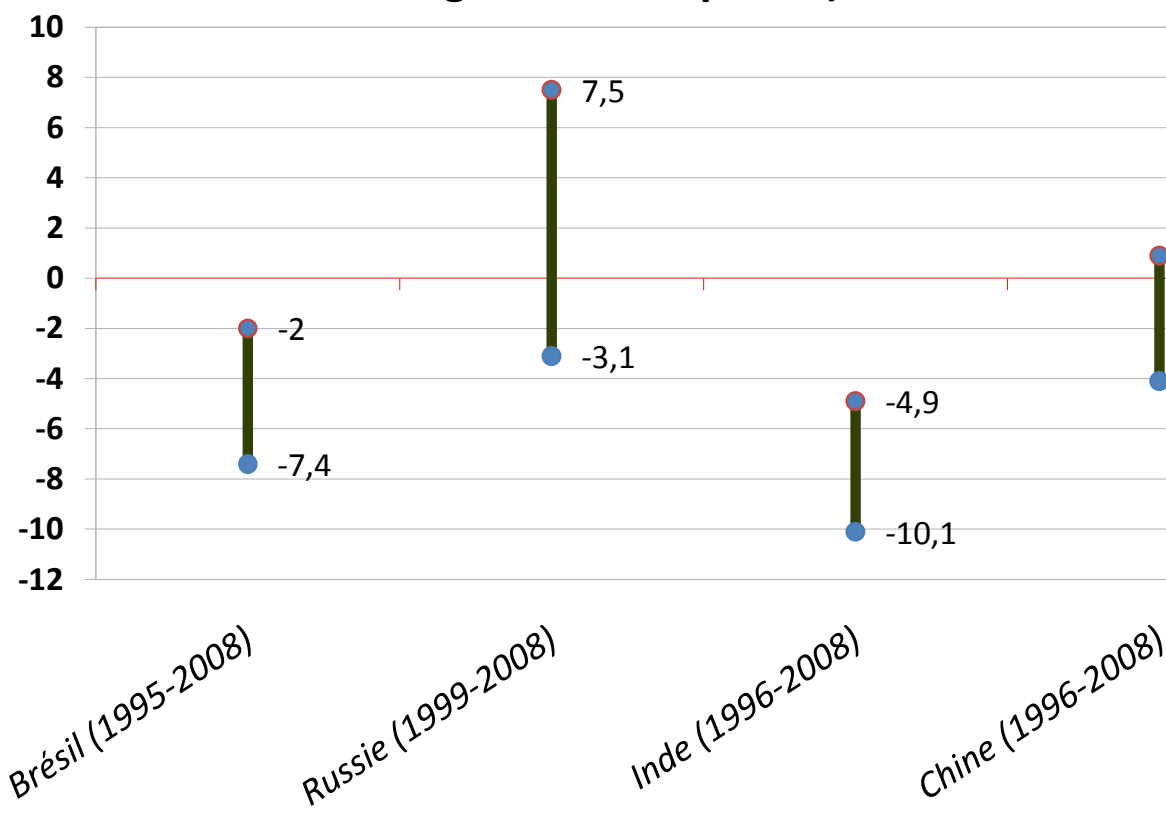

Source : IMF Country Report, années diverses. 


\section{Graphique 3. \\ Soldes comparés des transactions courantes (\% PIB, 1987-2008)}

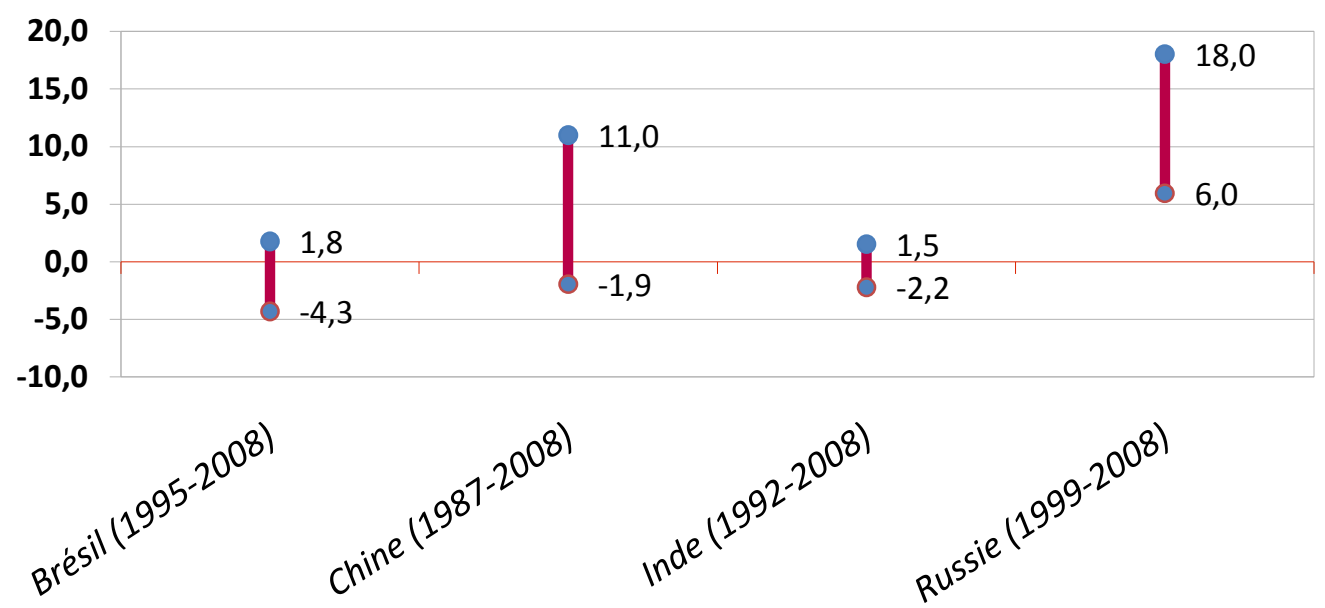

Source : IMF Country Report, années diverses.

Le processus d'émergence s'accompagne pour les quatre pays d'un taux d'inflation annuel de $6 \%$ en moyenne. La croissance, tirée en partie par la demande, est en elle-même inflationniste et les politiques monétaires des pays sont restées accommodantes, de manière à ne pas contrecarrer le dynamisme de l'économie. On remarque que l'inflation peut avoir atteint certaines années plus de $25 \%$, sans que la croissance soit remise en cause. La rigueur monétaire n'est donc pas l'apanage de l'émergence (Graphique 1).

Si l'on met à part le cas de la Russie, qui a profité de la manne pétrolière pour engager une politique de désendettement public anticipé, les économies étudiées s'accommodent aussi d'un déficit budgétaire consistant, situé en moyenne entre 2 et $4 \%$ du PIB, avec des pics pouvant dépasser $5 \%$ du PIB. Le Brésil et I'Inde n'ont pas connu d'excédent budgétaire depuis le début de l'accélération de leur croissance ; la Chine, une seule fois depuis 1996 (en 2007). Les grands pays émergents ne se caractérisent donc pas par leur orthodoxie budgétaire (Graphique 2).

La Russie et la Chine, pour des raisons différentes (les exportations chinoises bénéficient d'une excellente compétitivité prix, la Russie tire son excédent de ses exportations de matières premières, notamment énergétiques), sont bénéficiaires nets de leurs échanges de biens et services avec le reste du monde. L'Inde et le Brésil ne sont pas parvenus à équilibrer leur demande intérieure de biens et services étrangers par leurs exportations. L'émergence ne se traduit pas nécessairement par des excédents extérieurs (Graphique 3). 


\section{Conclusion (et troisième conjecture) : il n'existe pas de "one best way " de l'émergence économique}

Ainsi que nous le montrent les trajectoires des « BRIC », la diversité est caractéristique des processus d'émergence économique. A partir de situations structurelles différentes, ces quatre économiques présentent la caractéristique commune d'avoir engagé des réformes de grande ampleur dans leur système institutionnel (première conjecture). Mais leur rythme, leur profondeur et leurs conséquences n'ont pas été identiques et se sont avérées compatibles avec une certaine variété des choix de politique conjoncturelle. Les processus d'émergence ne nécessitent pas de se conformer à une doctrine économique unique (deuxième conjecture).

Cette diversité se retrouve dans les aspects plus structurels de l'insertion de ces économies dans la mondialisation: si, de ce point de vue, leur émergence a consisté notamment à tirer parti de l'intensification des échanges économiques mondiaux en captant une fraction croissante de la demande mondiale à leur profit, la manière d'obtenir ce résultat a été différente suivant les cas : la Russie, le Brésil ont pu s'appuyer sur la possession de ressources naturelles stratégiques qu'elles ont valorisées à l'international (insertion " malthusienne »); la Chine et l'Inde, pour une partie de leur développement industriel, sur leur capacité à fournir à moindre coût des biens à fort contenu en main d'œuvre par l'insertion des entreprises situées sur leur territoire dans les réseaux mondiaux de sous-traitance (insertion " ricardienne »); et pour certains secteurs de ces différentes économies, sur leur contribution au développement de nouveaux produits et services par la mise en œuvre d'une capacité d'innovation (insertion « schumpeterienne ») (Vercueil, 2011).

L'enseignement principal à retirer de cette rapide étude comparative est simple : il n'existe pas de modèle unique d'émergence que l'on puisse tirer de leur examen, pas de " one best way " qu'il serait possible de déduire de leur expérience au profit d'autres candidats à l'émergence. Au contraire, les BRIC nous semblent constituer une illustration convaincante de la variété des trajectoires possibles de croissance économique et d'insertion dans l'économie mondiale, pour des pays à revenus intermédiaires. 


\section{Références bibliographiques :}

Ahluwalia, M. S. (2002) : Economic Reforms in India since 1991 : Has Gradualism Worked ?, IBEF, http://203.122.59.38/download/msa008.pdf, consulté le 14/03/2011.

Alexashenko, S. (1992) : "General Remarks on the Speed of Transformation in the Socialist Countries", Review of Economies in Transition (BOFIT), n³, pp. 23-47.

Boyer, R. (2003) : "Les institutions dans la théorie de la régulation ", Cahiers d'économie politique, $n^{\circ} 44$.

Bresser-Pereira L. C. (2009): Mondialisation et compétition. Pourquoi certains pays émergents réussissent et d'autres non. Paris : La découverte.

Dunlop, J. B. (1999): "Shifting through the Rubble of the Yeltsin Years ", contribution to the Jamestown Institution Conference on Russia. Washington, D. C., mimeo.

Economic Commission for Europe (1992) : Economic Bulletin for Europe. Geneva : UNECE.

Favereau, O. (1989) : « Marchés internes, marchés externes », Revue économique, n40, pp. 121-189.

Feijo, Carmem, Punzo, Lionello F., Lamônica M. T. (2009) : "Brazil's economy 1971-2005: Growth pattern and structural change", CEDE discussion paper n ${ }^{\circ} 15,2009$, http://www.proac.uff.br/cede/.

IMF (2010) : World Economic Outlook - October. Washington, D. C. : International Monetary Fund.

Lin J. Y., Cai F., Li Z. (1996): The China Miracle. Development Strategy and Economic Reform. Hong Kong : The Chinese University Press, 1996.

North D. (1991) : "Institutions », Journal of Economic Perspectives, Vol. 5, n¹, Winter 1991, pp. 97112.

North (1997): "The Contribution of New Institutional Economics to the Understanding of the Transition Problem », United Nation / WIDER Annual Lecture, 7 march 1997.

Pagé J.-P., Vercueil J. (2004): De la chute du Mur à la Nouvelle Europe. Economie politique d'une métamorphose. Paris : L’Harmattan.

Roland G. (2000) : Transition and Economics. Politics, Markets, and Firms. Cambridge (Mass.) : the M.I.T. Press.

Stiglitz (1999) : "Whither Reforms ? Ten Years of the Transition », contribution to the Annual World Bank - IMF Conference on Development Economics. Washington, D. C. : 28-30 April 1999.

Vercueil (2002): Transition et ouverture de l'économie russe (1992-2002). Pour une économie institutionnelle du changement. Paris : L'Harmattan.

Vercueil (2010) : Les pays émergents. Brésil - Russie - Inde - Chine: mutations économiques et nouveaux défis. Paris : Bréal.

Vercueil (2011) : " S'immerger pour émerger. La singularité des modes d'ouverture des 'BRIC' ", in Piveteau A. (dir.), « Emergences économiques (titre provisoire) ». Paris : Karthala, à paraître. 